\title{
Thermal Conductivity Measurement of Graphene Composite
}

Jiuning $\mathrm{Hu}^{1,2}$, Wonjun Park ${ }^{1,2}$, Xiulin Ruan ${ }^{3,2}$, Yong P. Chen ${ }^{4,2,1}$

${ }^{1}$ School of Electrical and Computer Engineering, Purdue University, West Lafayette, IN 47907, U.S.A.

${ }^{2}$ Birck Nanotechnology Center, Purdue University, West Lafayette, IN 47907, U.S.A.

${ }^{3}$ School of Mechanical Engineering, Purdue University, West Lafayette, IN 47907, U.S.A.

${ }^{4}$ Department of Physics, Purdue University, West Lafayette, IN 47907, U.S.A.

\section{ABSTRACT}

Graphene composites (GCs) have attracted much attention recently. It is interesting to explore thermal properties of GCs in which graphene filler concentrations are tunable. Here, we use $3 \omega$ method to measure the thermal conductivity of GCs synthesized from reduced graphene oxide (RGO) dispersed in polystyrene. To avoid the detrimental effect of lithography processes to GCs, we have developed a novel method employing polyvinyl alcohol and poly(methyl methacrylate) (PMMA) as a holder film to transfer micrometer-sized metal heaters/sensors onto GC surface. Room temperature measurements of the thermal conductivity of GCs are performed. The thermal conductivity is enhanced by $\sim 35 \%$ when adding 5 vol. $\%$ of RGO filler concentration. Our measurements will be helpful to probe and understand the thermal transport properties of graphene based composites.

\section{INTRODUCTION}

Graphene composite (GC) materials have drawn much attention recently mainly due to its potential of large scale applications through mass production and abilities to tune their various properties by material engineering [1]. GCs exhibit excellent properties, such as extremely low percolation threshold of electrical conductivity [1]. Similarly, the thermal properties of GCs can be tuned by controlling graphene concentrations. It has been shown that adding graphene flakes to host materials, such as epoxy [2] and nanostructured phase change materials [3] can dramatically increase the thermal conductivity of the composite materials. However, there are few experiments to study the thermal transport in graphene-polystyrene composite which is inherently uniform through chemically compatible processes. It is interesting to investigate the thermal properties of such GCs, and in particular, the graphene concentration dependence of thermal conductivities.

\section{EXPERIMENT}

GCs of several different volume concentrations are made from polystyrene and graphene oxide [2]. Graphene oxide (GO) is produced by following Hummer's method [4]. The uniform mixture of $\mathrm{GO}$ and polystyrene in dimethylformamide is treated by hydrazine (Figure 1a) to convert GO to reduced GO (RGO, referred simply as graphene in the following text). The solution is then polymerized using methanol and filtered to collect $\mathrm{GC}$, followed by drying it in a 
vacuum oven. The dried GC is milled into fine powders (Figure 1b) and hot pressed into bulk dense composite plate as shown in Figure 1c. The thickness of the GC plate is about $1.3 \mathrm{~mm}$.

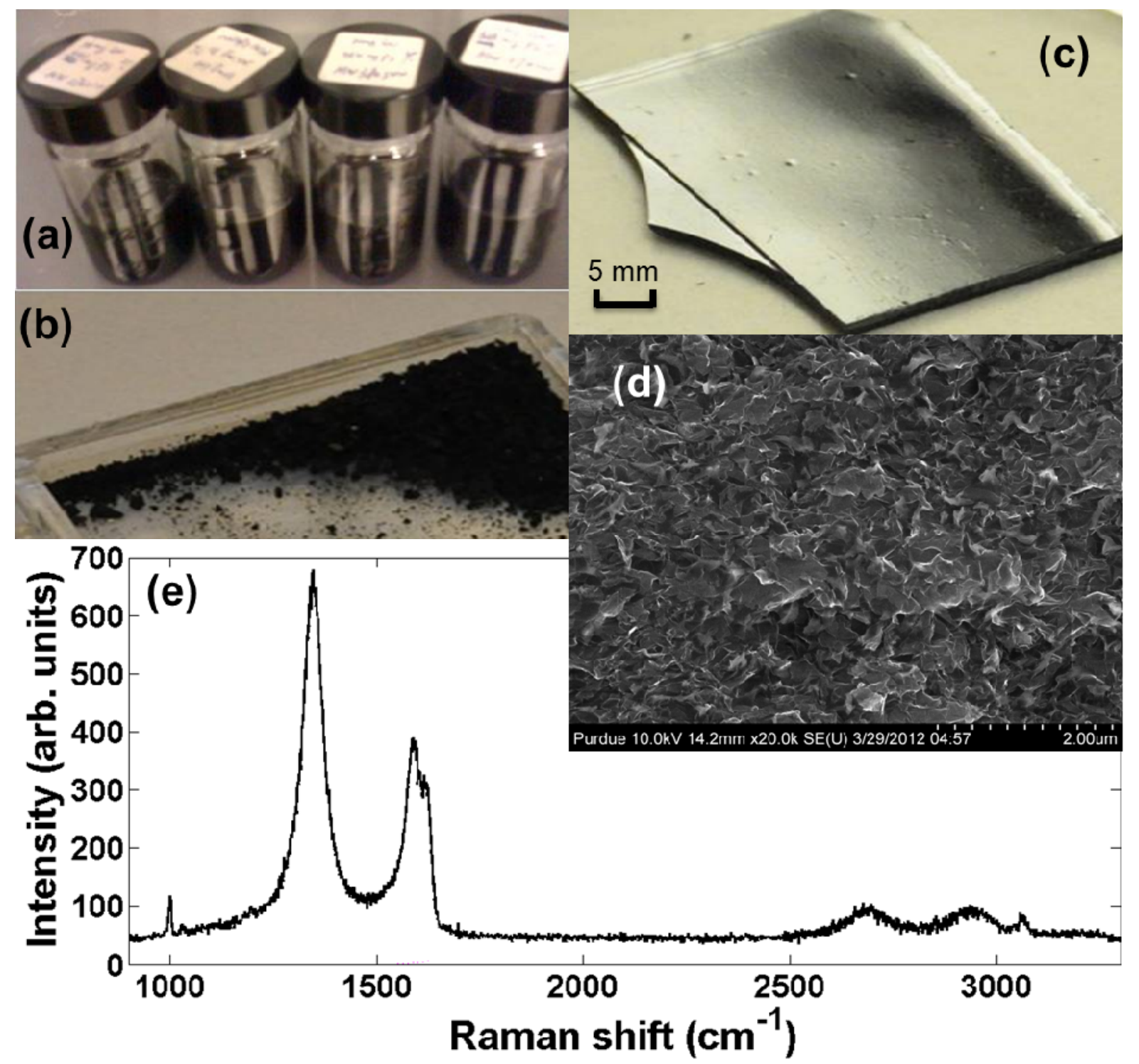

Figure 1. Main fabrication processes of graphene composite are shown in (a)-(c). The thickness of the hot pressed graphene composite plate in (c) is $1.3 \mathrm{~mm}$. The SEM image (d) and Raman spectrum (e) are provided.

The $3 \omega$ method [5] is employed to measure the thermal conductivity of GCs. To perform such measurement, metal lines ( $20 \mu \mathrm{m}$ wide and $500 \mu \mathrm{m}$ long) used as the heater and sensor need to be fabricated onto the GC surface using lithography techniques. GCs become soft and difficult to handle when they are submerged in acetone for a few minutes or longer. In order to avoid this detrimental effect during lithography processes such as lift-off, a novel transfer method is developed to place the metal line on the surface of GCs. The transfer processes are shown in Figure 2. We start from a piece of silicon substrate covered with polyvinyl alcohol 
(PVA) film of $\sim 0.5 \mu \mathrm{m}$ thick (Figure 2a). The standard electron beam lithography is used to fabricate metal lines with contact pads on top of the PVA layer (Figure 2b) and subsequently the substrate is coated with a thin layer of poly(methyl methacrylate) (PMMA) of $\sim 0.3 \mu \mathrm{m}$ thick. The substrate is then placed in a water bath to remove the PVA layer (Figure 2d) and the PMMA layer with metal lines is floating on the surface of the water bath as shown in the red dashed circle in Figure 2g. The PMMA layer is then transferred onto the surface of GCs (Figure 2e and $2 \mathrm{~h}$ ) and washed in acetone for 5 seconds to remove the PMMA layer (Figure 2f). It is worthy to note that washing GCs in acetone for 5 seconds won't damage GCs, but most of the PMMA layer is stripped off to facilitate the wire bonding. The metal lines are finally bonded onto a chip carrier to perform measurement (Figure 2i).

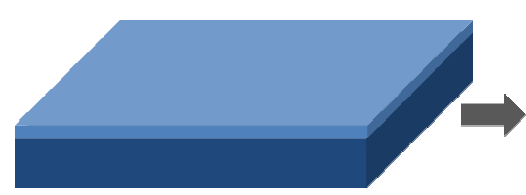

(a)

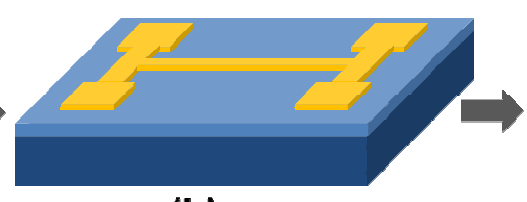

(b)

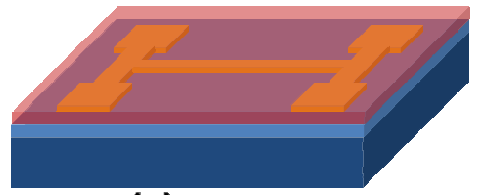

(c)

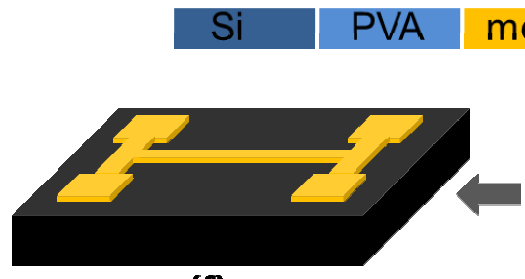

(f)

\section{metal PMMA GC}

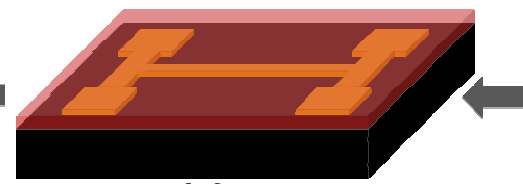

(e)

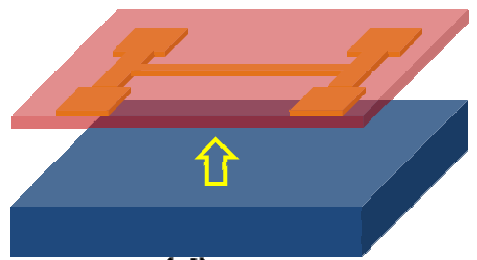

(d)

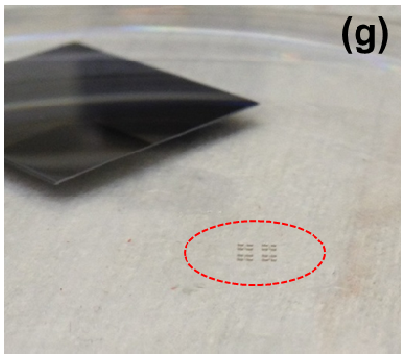

(g)

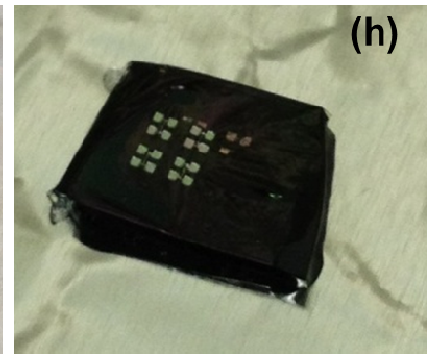

(h)

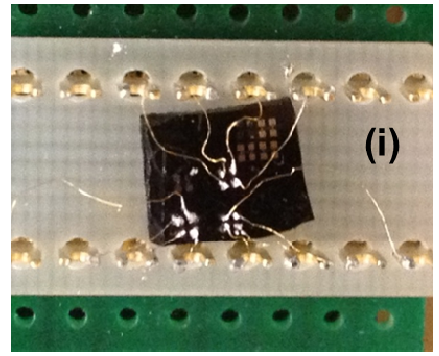

Figure 2. The metal line is transferred onto GC surface as shown in cartoons (a)-(f). After dissolving PVA in the water bath, the PMMA layer with metal line is at the surface of the water bath, indicated by the dashed circle in (g). In (h), the metal line with contact pads is transferred onto the GC surface. Figure $2 \mathrm{i}$ shows the wire bonded sample mounted on a chip carrier.

During the measurement, first, the temperature coefficient of the metal line is measured for calibration in later data fitting. The GC sample in Figure $2 \mathrm{i}$ is then placed in a vacuum stage with pressure typically less than $10^{-5}$ Torr. All of the measurements are done around room temperature. An electrical bridge shown in Figure 3 is used to cancel the dominant $1 \omega$ signal to increase the $3 \omega$ signal to noise ratio. The variable resistor is adjusted to match the resistance of the metal line, so that the $1 \omega$ component $V^{\prime}{ }_{1 \omega}$ of the output of the lock-in amplifier is brought close to zero. The $3 \omega$ voltage $V_{3 \omega}$ (amplitude value) is then taken over the frequency range of 10 $-1000 \mathrm{~Hz}$. 


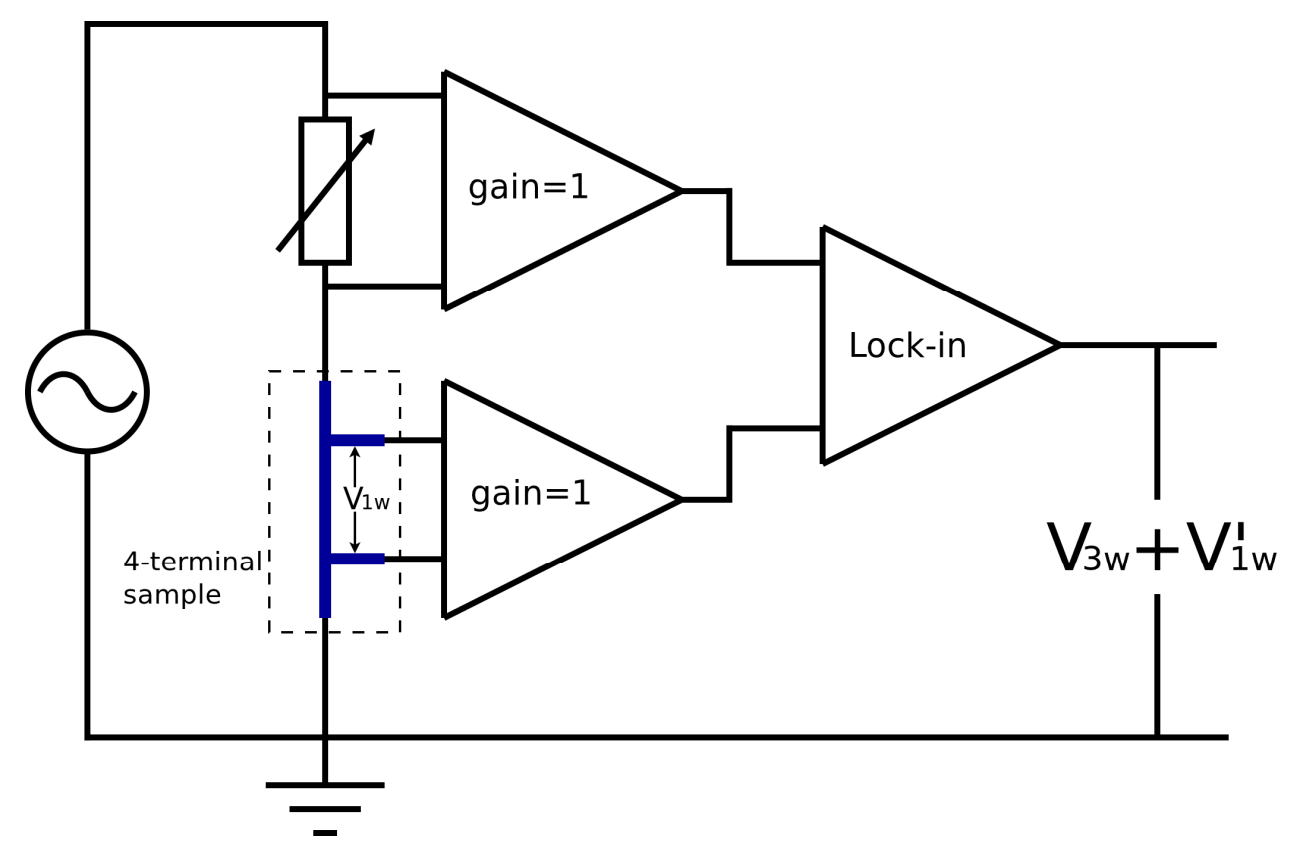

Figure 3. Electrical bridge used to measure the $3 \omega$ signal.

\section{RESULTS AND DISCUSSIONS}

Our GCs are characterized firstly by scanning electron microscopy (SEM) to study its uniformity. As shown in Figure 1d for a GC sample of 5 vol.\%, graphene is uniformly dispersed in the polystyrene host. Graphene flakes form the backbone of the umbrella structure in the SEM image [1]. The Raman spectrum of a GC sample of 5 vol.\% exhibits typical peak signatures of chemically reduced graphene oxide [6].

To calculate the thermal conductivity of GCs, $V_{3 \omega}$ is firstly converted to the thermal impedance

$$
Z(\omega)=-\frac{4 V_{3 \omega}}{I_{1 \omega} V_{1 \omega}^{2} \beta}
$$

where $I_{l \omega}$ is the amplitude of the $1 \omega$ current component flowing in the metal line, $V_{l \omega}$ is the amplitude of the voltage drop of $1 \omega$ component along the metal line (Figure 3 ) and $\beta$ is the temperature coefficient of the metal line. The temperature coefficient satisfies the relation $R(T)=R\left(T_{0}\right)\left[1+\beta\left(T-T_{0}\right)\right]$ where $R(T)$ is the temperature dependent resistance of the metal line. Since the thickness of the composite is much larger than the thermal wave penetration depth in the frequency range of $10-1000 \mathrm{~Hz}$, it is safe to approximate the metal line as sitting on a semi-infinite GC, and the thermal impedance can be expressed as

$$
Z(\omega)=\frac{1}{\pi L \kappa a^{2}} \int_{0}^{\infty} d l \frac{\sin ^{2} l a}{l^{2} \sqrt{l^{2}+i 2 \omega C / \kappa}}
$$


where $L$ is the length of the metal line, $a$ is the half-width of the metal line, $C$ is the product of mass density and specific heat capacity of GCs, and $k$ is the thermal conductivity of GCs [7]. Equation 2 is used to fit two parameters $C$ and $k$.

Figure 4 shows the measurement results for a 5 vol.\% sample. From the resistance vs. temperature curve (Figure $4 \mathrm{a}$ ), the fitted temperature coefficient for the metal line on the 5 vol.\% GC sample is $\beta=33.7 \times 10^{-3} 1 / \mathrm{K}$. The thermal impedance is complex, and its in-phase (real part) and out-of-phase (imaginary part) components are separately plotted in Figure $4 \mathrm{~b}$. The fitted thermal conductivity for the 5 vol. $\%$ sample is $0.183 \pm 0.020 \mathrm{~W} / \mathrm{m}-\mathrm{K}$. The fitted product of mass density and specific heat capacity of the 5 vol.\% sample is $C=1.52 \pm 0.23 \times 10^{6} \mathrm{~J} / \mathrm{K}-\mathrm{m}^{3}$. Since only a small amount of graphene is added, we can use the mass density of $1.05 \mathrm{~g} / \mathrm{cm}^{3}$ [1] of polystyrene used in our experiment. The specific heat capacity of the 5 vol.\% sample is then $c_{p}=1.45 \pm 0.22 \mathrm{~J} / \mathrm{K}-\mathrm{g}$. The pure polystyrene we measured has a thermal conductivity of $0.136 \pm 0.020 \mathrm{~W} / \mathrm{m}-\mathrm{K}$ which is very close to the reported value of $0.14-0.15 \mathrm{~W} / \mathrm{m}-\mathrm{K}[8,9]$. The fitted product of mass density and specific heat capacity of pure polystyrene is $\mathrm{C}=1.69 \pm 0.25 \times 10^{6}$ $\mathrm{J} / \mathrm{K}-\mathrm{m}^{3}$. Given the mass density of $1.05 \mathrm{~g} / \mathrm{cm}^{3}[1]$ of polystyrene, its specific heat capacity is $c_{p}=1.61 \pm 0.24 \mathrm{~J} / \mathrm{K}-\mathrm{g}$, close to the reported value of $\sim 1.2 \mathrm{~J} / \mathrm{K}-\mathrm{g}$ [12]. The thermal conductivity is increased by $\sim 35 \%$ after adding graphene with volume percent of 5 vol. $\%$. Compared to the recent experiment on a much larger enhancement of thermal conductivity of 5 vol.\% mechanically exfoliated graphene and epoxy composite [2], we have much less enhancement. One reason may be due to the poor quality of chemically reduced graphene oxide with typical thermal conductivity less than $10 \mathrm{~W} / \mathrm{m}-\mathrm{K}[10,11]$ which is much less than that of high quality exfoliated graphene.

Our transferred metal sensor has a good thermal contact with the composite sample. This can be justified by estimating the thermal boundary resistance between the sensor and the composite samples. We add a constant term $Z_{b}$ to equation (2) to consider the thermal boundary resistance. When $Z_{b}$ is increased to about $100 \mathrm{~K} / \mathrm{W}$, the fitted curve in Figure $4 \mathrm{~b}$ starts to deviate from the measured curve appreciably. We can conclude that the boundary thermal impedance $\mathrm{Z}_{\mathrm{b}}<100 \mathrm{~K} / \mathrm{W}$ (corresponding to a thermal boundary resistance of about $1 \mathrm{~mm}^{2} \mathrm{~K} / \mathrm{W}$ ) which is negligible compared to the typical measured thermal impedance values that are usually larger than $1000 \mathrm{~K} / \mathrm{W}$. 

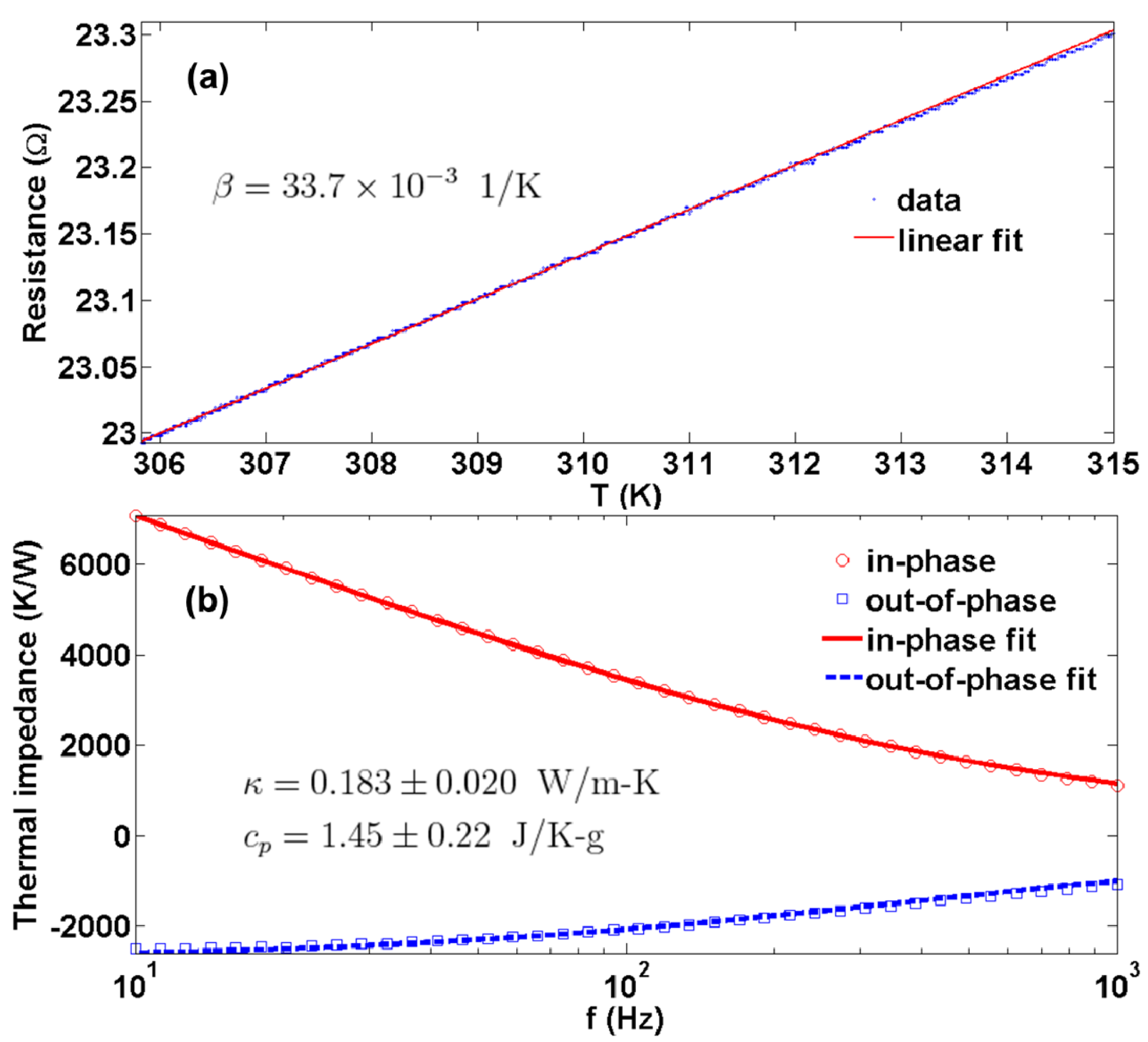

Figure 4. Resistance vs. temperature (a) for metal line on the 5 vol.\% (graphene filling) sample and the measured thermal impedance vs. frequency (b) for the 5 vol. $\%$ sample.

\section{ACKNOWLEDGMENTS}

This work is partially supported by the Semiconductor Research Corporation (SRC) Nanoelectronics Research Initiative (NRI) via Midwest Institute for Nanoelectronics Discovery (MIND) and the Cooling Technologies Research Center (CTRC).

\section{REFERENCES}

1. S. Stankovich, D.A. Dikin, G.H.B. Dommett, K.M. Kohlhaas, E.J. Zimney, E.A. Stach, R.D. Piner, S.T. Nguyen and R.S. Ruoff, Nature 442, 282 (2006).

2. K.M.F. Shahi and A.A. Balandin, Nano Lett. 12, 861 (2012). 
3. F. Yavari, H.R. Fard, K. Pashay, M.A. Rafiee, A. Zamiri, Z. Yu, R. Ozisik, T. Borca-Tasciuc and N. Koratkar, J. Phys. Chem. C 115, 8753 (2011).

4. W.S. Hummers Jr. and R.E. Offeman, J. American Chemical Society 80, 1339 (1958).

5. D.G. Cahill, Rev. Sci. Instrum. 61, 802 (1990).

6. D.J. Late, U. Maitra, L.S. Panchakarla, U.V. Waghmare and C.N.R. Rao, J. Phys.: Condens. Matter 23, 055303 (2011).

7. C. Dames and G. Chen, Rev. Sci. Instrum. 76, 124902 (2005).

8. S. Yu, P. Hing and X. Hu, Composites: Part A 33, 289 (2002).

9. J. Chiu and P.G. Fair, Thermochim. Acta 34, 267 (1979).

10. W. Yu, H. Xie, X. Wang and X. Wang, Physics Letters A 375, 1323 (2011).

11. T. Schwamb, B.R. Burg, N.C. Schirmer and D. Poulikakos, Nanotechnology 20, 405704 (2009).

12. Y.P. Koh, G.B. Mckenna and S.L. Simon, Journal of Polymer Science Part B: Polymer Physics 44, 3518 (2006). 